\title{
Lasertherapy as A Strategy for Treatment Healing under Caloric Restriction - Study in Rats
}

Leandro C. Moscardi, Talita P. Espíndola, Amanda A. Ferreira, Naiara Alves, Maria Esméria C. Amaral, Andréa A. Aro, Rodrigo A. Dalia, Marcelo A. M. Esquisatto, Fernanda A. S. Mendonça, Gláucia M. T. Santos and Thiago A. M. Andrade

Graduate Program in Biomedical Sciences of the Herminio Ometto University Center, UNIARARAS, Araras, São Paulo 13607-339, Brazil

\begin{abstract}
Complications in the healing process are challenging, especially in clinical situations of caloric restriction (CR). The lasertherapy becomes an important strategy that aids the repair, especially in CR. Thus, it is important to investigate the InGaAlP-660 $\mathrm{nm}$ laser as an strategy to repair cutaneous wounds in rats submitted to $30 \%$ of CR and to understand the tissue repair in clinical situations of CR. Thirty-six male Wistar rats were used, of which half were fed with $30 \%$ less ration, and half with ad libitum diet, for 21 days. Then, punch lesions of $1.5 \mathrm{~cm}$ in diameter were made on the animals backs, which were divided into: NR (no-restricted), R (restricted)—both before lesion; C (control), RC (restricted-control), L (laser), RL (restricted-laser)—after lesion. Samples of the skin/lesion/scar were collected on the 2nd, 7th and 14th days post-injury for histological, biochemical and molecular analyses. The $\mathrm{R}$ group showed reduction of body mass, epidermal/dermal thickness, inflammation, angiogenesis, fibroplasia and collagenesis. The RL group showed control of inflammation, oxidative damage and increase of antioxidants than RC, which probably favored angiogenesis, collagenesis and reepithelialization, similar to C and L. Thus, 30\% of CR impaired the skin (before lesion). In the lesion, lasertherapy has shown to be effective in tissue repair mainly in CR status, being thus, the laser clinically important strategy to tissue repair in critical situations of caloric restriction.
\end{abstract}

Key words: Wound healing, skin, caloric restriction, wound treatment, therapeutic strategy, lasertherapy.

\section{Introduction}

Wound healing involves celular events, cytokines and growth factors production on wound, aiming the complete reepithelialization. In inflammatory phase, mediators promote chemotaxis, mainly of neutrophils and macrophages, which are involved in tissue debridement and development of oxidative stress [1]. This is characterized by imbalance between oxidants and antioxidants, due to the excessive production of ROS (reactive oxygen) and RNS (nitrogen) species and the reduced neutralization of these by the antioxidants. Then, oxidative damage is aggravated, mainly when associated with caloric restriction (CR),

Corresponding author: Thiago Antônio Moretti de Andrade, Ph.D., research fields: immunophysiopathology of healing using new therapeutics in differents experimental models. causing difficulties on tissue healing [2]. Moreover, angiogenesis improves nutritional and oxygen supply in the wound bed. The collagen and extracellular matrix production by fibroblasts recompose the damaged tissue, promoting the reepithelialization [3].

In this view, it is noted the healing process requires adequate nutritional status, since it consumes a good part of the body's reserve. In CR, the level of nutrients provided is comparable to ad libitum consumption, but with a reduction in calories consumed, i.e., undernutrition without malnutrition [4].

Moreover, there are impacts of $\mathrm{CR}$ on the immune system [5] and, consequently, on the wound healing, because the immune system is expensive in terms of demands for energy [6] being it the first systems whose sacrifices might be made at the beginning of CR [5]. In these ways, CR was found to have adverse 
effects on wound healing [7] and resulted in decreased of fibroblasts function and collagen accumulation [8], limiting the granulation tissue.

The sirtuin (SIRT) regulates activity of many proteins that are related to energy metabolism, cell survival and longevity [9]. CR increases the expression of SIRT-1 in multiple tissues and it is increased in response to nutrient deprivation in cultured cells [10]. SIRT-1 controls the gluconeogenic/glycolytic pathways in the liver in response to fasting or food restriction [11]. Moreover, SIRT activation by $\mathrm{CR}$ has been implicated in changes in the immune system, because the immune activation is energetically expensive [12]. It has been shown that activation of the immune system significantly increases mortality in nutrient-limited settings [13]. Thus, the inhibitory effect of SIRT-1 on $\mathrm{NF}-\mathrm{kB}$ activity is consistent with its role in regulating the available nutrients during times of energy crisis, diverting calories away from the immune system to spare them for essential survival processes [12], which probably can cause deficit in the healing process.

In this context, there is an intense difficulty in the treatment of some types of cutaneous wounds, especially when associated with nutritional scarcity. Low-intensity lasertherapy has been noted for its important healing activity [14]. Moreover, there is evidence that laser has been shown important healing effects on tissues due to its physiological and therapeutic effects depending on the absorption of photons by chromophores in the target tissue. Studies have shown that the photons emitted by the laser are transformed in ATP and used by the cell to power needed metabolic activities, such as cell proliferation, and collagen synthesis. All of them, accelerate the tissue repair in chronic wounds [15, 16], especially when associated with oxidative stress status, like nutritional deficit and clinical situations of $\mathrm{CR}$, for example. In this view, it was hypothesized that wounds associated with $30 \%$ of CR are healing slowly or not at all, may benefit from lasertherapy.

Cardiovascular diseases, diabetes, cancer, inflammation, chronic wounds and neurodegeneration disease are examples of human pathologies, directly or indirectly affected by amount of nutrients [17]. Furthermore, patients bedridden by these and/or other pathologies, when presenting cutaneous lesions, either from post-surgical or pressure wounds, will be difficult to heal due to the nutritional scarcity caused by these pathologies and/or poor nutrition. And this, probably could be the particular clinical situation that lasertherapy is more effective. Thus, it is important to investigate the laser (InGaAlP-660 nm) as an strategy to repair cutaneous wounds in rats submitted to $30 \%$ of $\mathrm{CR}$ and to understand the tissue repair in clinical situations of CR.

\section{Material and Methods}

\subsection{Animals}

All surgical and experimental procedures used in this study were conducted according to the experimental requirements and biodiversity rights of the National Institutes of Health for the Care and Use of Laboratory Animals ((NIH Publication 80-23, reviewed in 1996). Studies were performed according to the norms established by the Arouca Law, approved by the ethical principles in animal research adopted by COBEA (Brazilian College of Animal Experimentation) and approved by the Ethics Commission on Animal Use (CEUA) of the Herminio Ometto University Center (UNIARARAS) protocol $n^{\circ}$ 021/2015.

Thirty-six male Wistar rats (Rattus norvegicus albinus) within 90 days of age, obtained from the Center of Animal Experimentation "Prof. Dr. Luiz Edmundo de Magalhães" from the UNIARARAS (Herminio Ometto University Center) were used. The animals were housed in individual polycarbonate cages under constant temperature conditions $(23 \pm$ $2{ }^{\circ} \mathrm{C}$ ), humidity (55\%) and light/dark 12:12 hours 
cycle with free access to commercial standard ration and water for the entire experiment period.

\subsection{Experiment Design}

2.2.1 Experimental Model of 30\% of Caloric Restriction.

The ration was daily weighed for five days to evaluate the animals intake-average on ad libitum diet. For CR establishment, it was offered to 18 animals ( $R$ group-before lesion $=\mathrm{RC}$, and $\mathrm{RL}$ groups, after lesion) $30 \%$ less ration from the intake-average, during 21 days. The others 18 rats were the control groups (NR groups - before lesion $=\mathrm{C}$, and L groups, after lesion), which remained on ad libitum diet during 35 days of experiment.

On the 21st day of CR, wounds were performed on the all animals dorsum, which have been followed-up by 2 nd, 7 th and 14 th days ( $n=3$ rats/group/time of follow-up) [18].

\subsubsection{Caloric Restriction Confirmation}

The blood samples were collected directly from the heart-left ventricle of last euthanasia on $14^{\text {th }}$ day post-injury (35th day of $\mathrm{CR}$ ). Serums were subsequently stored at $-20{ }^{\circ} \mathrm{C}$ for analysis of glucose, cholesterol, triglycerides, protein and albumin according to the manufacturer's standard instructions (Laborlab, Guarulhos-SP-Brazil). In addition, the animals were weighed on 1st, 7th, 14th, 21st and 35th days of CR (35th day of CR was similar to 14th day post-injury) and also analyzed for SIRT-1 expression by Western blotting [18].

\subsubsection{Surgical Procedure and Experimental Groups}

The rats were anesthetized by the combination of ketamine $(3.0 \mathrm{~mL} / \mathrm{kg})$ with xylazine $(1.0 \mathrm{~mL} / \mathrm{kg})$ for skin injuries performance. Then, trichotomy was performed on the skin of the dorsum of all animals. For a greater use (refinement) and a reduction on the animal's amount, two excisions were performed on the dorsal region of each rat using a histological punch of $1.5 \mathrm{~cm}$ diameter, reaching the dermo-epidermal region until the exposure of the muscular fascia $[16$,
19-21]. These both skin samples obtained were used to investigate the skin changes caused by $30 \%$ of CR in the skin before lesion (without treatment), obtaining the groups $(n=6$ animals/group $)$ NR (no-restricted-on ad libitum diet) and $\mathrm{R}$ (restricted - under $30 \%$ of CR).

After performing the two wounds, all animals were divided into four distinct experimental groups (each group with $n=3$ animals/group/time of follow-up): C (control: wounds treated topically with laser switched off and ad libitum diet); RC (restricted-control: treated with laser switched off and 30\% of CR); L (laser: wounds with topically lasertherapy and ad libitum diet); RL (restricted-laser: lasertherapy and $30 \%$ of $\mathrm{CR}$ ).

Then, both lesions from each rat received the same specific treatment and no occlusive dressing was used. The animals were placed in individual cages and received 10 drops of analgesic (dipyrone) diluted in $250 \mathrm{~mL}$ of water for $72 \mathrm{~h}$.

\subsubsection{Lasertherapy}

It was used Photon Lase III (DMC ${ }^{\circledR}$-São Carlos-SP-Brazil) InGaAlP (indium-gallium-aluminum-phosphor): 660 $\mathrm{nm}$ wavelength (visible-red), $3.63 \mathrm{~W} / \mathrm{cm}^{2}$ of irradiance, $100 \mathrm{~mW}$ of power, $134.54 \mathrm{~J} / \mathrm{cm}^{2}$ of energy density, 3.7 $\mathrm{kJ}$ of energy/point, $14.8 \mathrm{~J}$ of total energy dose, 0.0275 $\mathrm{cm}^{2}$ of beam covering area application, 37 seconds under continuous mode in 4 points at the wound extremities, $\pm 2.0 \mathrm{~mm}$ of distance from each wounds by $90^{\circ}$ of angle between wound and applicator probe. The treatments were started soon after the experimental wound and performed $3 \times /$ week (at the same time).

\subsubsection{Sample Harvesting}

After 2nd, 7th and 14th days post-wound, the animals were euthanized with anesthetic deepening (ketamine: $3.0 \mathrm{~mL} / \mathrm{Kg}$ and xylazine: $1.0 \mathrm{~mL} / \mathrm{Kg}, 300$ $\mu \mathrm{L} / \mathrm{Kg}$ animal body weight) and cervical dislocation. Then, both skin/wound/scar were harvested and divided in parts for histologicals, biochemicals and moleculars studies $(n=3$ part of wound/scar of 
rats/group/time of follow-up).

\subsubsection{Evaluation of Wounds Reepithelialization}

On the $0,2 \mathrm{nd}, 7$ th and 14 th days, both wounds of each animal were photographed in basic mode, without flash, without zoom and using the same camera. To standardize the distance from the camera to the wound, the camera was attached to a support 30 $\mathrm{cm}$ apart and perpendicular to the rat wound. A ruler, arranged beside the animal and along the wounds, was used to standardize the unit area of the wounds in 2 $\mathrm{cm}$ and also to serve as a known measure in the calibration of the ImageJ software when calculating the area of the wound.

ImageJ software was used to determinate the wound area to calculate the Wound Healing Rate: WHR (wound healing rate) $=($ initial area - final area $) /$ initial area. The initial area corresponds to the day of the surgical procedure ( 0 day) and the final area corresponds to the day of euthanasia (2nd, 7 th or 14th days, post-injury). WHR values greater than zero represent a decrease in wounded area (reepithelialization) and smaller than zero represent an increase in wounded area [16, 20].

\subsubsection{Histomorphometry analysis}

The skin/wound/scar $(n=3 /$ group/time of follow-up) biopsies were conditioned for 48 hours in $10 \%$ buffered formaldehyde solution and followed by paraffin-histological processing (Hematoxylin-Eosin) for simultaneous quantification of inflammatory infiltrate, fibroblasts and blood vessels using the "Cell Counter" Plugin of ImageJ software. It was used 5 images (magnification: 400×) and the quantification of inflammatory infiltrate, fibroblasts and blood vessels were double-blind. Results were expressed as number of cells' average [21].

For measuring epidermal and dermal thickness, histological images $(400 \times$ of magnification) were taken in sequence from the epidermis to the subcutaneous cellular tissue. These images were automatically grouped using the Photomerge function of the Adobe Photoshop CC 2018. By the ruler on
Photoshop, 3 lines/image was traced on epidermis and dermis and then, Photoshop supplied the thickness (cm) [16].

\subsubsection{Myeloperoxidase (MPO)}

The MPO assay was performed for the determination of the neutrophilic infiltrate by the macerate supernatant samples of the skin/wound/scar of the rats during the follow-up and treatments. Samples were homogenized by Polytron $^{\circledR}$ homogenizer (Kinematica, USA). After centrifugation, $5.0 \mu \mathrm{L}$ of supernatant was diluted in $45 \mu \mathrm{L} 0.08 \mathrm{M}$ $\mathrm{NaPO}_{4}$. MPO activity was detected using $25 \mu \mathrm{L} 1.6$ $\mathrm{mM}$ tetramethylbenzidine (Sigma-Aldrich $\left.{ }^{\circledR}, \mathrm{USA}\right)$ and read at $450 \mathrm{~nm}\left(\right.$ SpectraMax $^{\circledR}$, USA) [21].

\subsubsection{N-Acetylglicosaminidase (NAG)}

NAG assay was performed to macrophagic infiltration. It was placed $3.0 \mu \mathrm{L}$ of supernatant from the macerated samples and it was added $30 \mu \mathrm{L}$ p-nitrophenyl-2-acetamide- $\beta$-D-glucopyranoside (Sigma-Aldrich), diluted in $50 \mu \mathrm{L}$ of $50 \mathrm{mM}$ citrate buffer. Finally, $50 \mu \mathrm{L}$ of $0.2 \mathrm{M}$ glycine was added and read at $405 \mathrm{~nm}$ [22].

\subsubsection{Hydroxyproline (HO-Pro)}

HO-Pro assay was performed to total collagen determiantion. After hydrolysis in $6 \mathrm{~N} \mathrm{HCl}$ at $110{ }^{\circ} \mathrm{C}$, the samples were treated with $1.41 \%$ chloramine $\mathrm{T}$ and $15 \%$ p-dimethylaminobenzaldehyde, followed by incubation at $60^{\circ} \mathrm{C}$ and read at $550 \mathrm{~nm}$ [23].

\subsubsection{Western blotting}

Samples were homogenized, treated with Laemmli buffer and coursing on SDS-PAGE (10\% to $12 \%$ Tris-acrylamide) in minigel apparatus (Miniprotean). The proteins were transferred to PVDF (polyvinylidene difluoride) membrane (Immun-Blot ${ }^{\circledR}$ Bio-Rad), which were incubated overnight with: 1:200 anti-TGF- $\beta 1$, anti-CAT, anti-SOD1, anti-SOD2, anti-VEGF, $\beta$-actin (Santa Cruz Biotechnology-USA); 1:500 anti-collagen I (SIGMA) and, 1:1,000 anti-SIRT-1 (CellSinaling, USA) and 1:2,000 anti-collagen III (Santa Cruz Biotechnology-USA). The membrane was incubated with 
chemiluminescence antibodies kit and revealed in Syngene G:BOX-photodocumentator. The bands' intensity was evaluated by densitometry using ImageJ [18].

\subsubsection{Oxidative stress and antioxidants}

The lipoperoxidation products were analyzed by measuring the formation of thiobarbituric acid reactive species (TBARS) [24]. Then $100 \quad \mu \mathrm{L}$ tissue homogenates was deproteinized with $20 \%$ trichloroacetic acid. The supernatant was exposed to $0.7 \%$ thiobarbituric acid, heated to $95{ }^{\circ} \mathrm{C}$ and read at $535 \mathrm{~nm}$.

The -SH groups (antioxidant) were quantified by the reaction of the sulfhydryl group with 5,5' dithiobis (2-nitrobenzoic acid) (DTNB) and read at $412 \mathrm{~nm}$ [25].

\subsection{Statistical Analysis}

Data were expressed as mean \pm standard error. All data were passed for Kolmogorov-Smirnov normality test. For the data fitting the normality curve, the statistical test chosen was $t$-Student test for before lesion groups (comparing NR/R groups) and ANOVA Two-Way (Multiple-comparisons) and Tukey post-test to groups after lesion (comparing $\mathrm{C} / \mathrm{RC} / \mathrm{L} / \mathrm{LR}$ groups). For the data not fitting in the normality curve (glucose/cholesterol/protein/albumin) the statistical test was Mann-Whitney (for triglycerides was $t$-Student test), all were performed in Statistic 7.0 and GraphPad Prism 6.0 softwares. The pre-established level of significance was $p<0.05$.

\section{Results and Discussion}

\subsection{Caloric Restriction Status and Implications on Healing}

Nutritional deficiency remains one of the most common causes of morbidity and mortality among children, also prevalent among elderly people [26] and hospitalized patients specially in underdeveloped countries. Even in industrialized countries, people are vulnerable to protein malnutrition, which can accompany weight control, lifestyle-related disease and aging. Diets in these populations are frequently deficient in macronutrients, micronutrients, or both [27-29]. The nutritional deficit is usually found in these at-risk populations and is a critical factor in susceptibility to infection [30]. Numerous nutrients are known to be important for optimal wound healing. $\mathrm{CR}$ is associated with immunological changes, making wound healing inadequate due to inflammatory phase prolongation and fibroplasia impairment, reduction in collagen and extracellular matrix synthesis, angiogenesis and wound remodeling [31, 32].

As a treatment of these immunocompromised wounds, lasertherapy has been an important alternative because the phototherapy depends on the absorption of photons by chromophores in the target tissue, accelerating the tissue repair [15] mainly as in pathological conditions such as in CR status. Then, wounds associated with nutritional scarcity heal slowly or not at all, and the lasertherapy could benefit the treatment in a $30 \%$ of CR animal model. In this way, it becomes relevant to investigate the effects of lasertherapy as a strategy in the repair of cutaneous lesions in rats submitted to $30 \%$ of caloric restriction.

To confirm CR-status, $\mathrm{R}$ group showed important reduction of body weight relation to NR from 7 th to 35th days of CR. Only NR presented important increase of body mass during the follow-up of $\mathrm{CR}$ relation to previous days (Fig. 1A). This finding corroborates with Hunt et al. [4] on $40 \%$ of CR.

About the biochemical analyses, there was no difference between NR and R for glucose, protein and albumin (Fig. 1C, 1F and 1G) on the 35th day of CR. However, about cholesterol and triglycerides (Fig. 1D and $1 \mathrm{E})$ the $R$ group was lower than NR, corroborating to Amaral et al. [18]. Low body weight, hypocholesterolemia and hypotriglyceridemia contents are features usually found in animals submitted to $\mathrm{CR}$, indicating the adequacy of the animal model used in this work [18]. The Caloric-Restriction-Society/CRS consists of a group 
of humans individuals who practice CR with optimum nutrition (CRON). The subjects of the study were 18 volunteers (the CRONies) that have been practicing the restricted-diet for 3-15 years. Classical signs of $\mathrm{CR}$ adaptations were evident in the CRONies, showing significantly lower blood glucose levels, corroborating to our results, insulin levels, and blood pressure. Lipid profiles of the CRONies were significantly healthier than the height-matched controls. Specifically, total cholesterol, LDL, and triglycerides were all reduced, corroborating to our results, and HDL was elevated compared to controls [33]. Regarding to our similar results of serum protein and albumin between both groups, it is possibly due to the slow and late reduction in CR [34].

$\mathrm{CR}$ induces the expression of SIRT-1 that participates as signaling of nutritional scarcity [35]. Serum was collected from humans after CR increased SIRT-1 expression. Among the changes related to SIRT-1 are increased hepatic gluconeogenesis, decreased glycolysis and increased hypothalamic signaling of hunger [36]. In our results SIRT-1 was increased, mainly on 7th and 14th days and in LR its expression was superior to the other groups (Fig. 1B). In addition, the increase of SIRT-1 in the L and LR compared to $\mathrm{C}$ and $\mathrm{CR}$ on 14th day, might suggest laser associated with $\mathrm{CR}$ can increase SIRT-1 expression on tissues. Besides SIRT-1 being a CR marker, it has shown SIRT-1 is required for efficient wound healing when it was used on mice with epidermis-specific SIRT-1 deletion. SIRT-1 deficiency in this model inhibits the repair of epidermis and dermis, altering the production of many cytokines, inhibited the recruitment of macrophages, neutrophils and mast cells, increased oxidative stress and reduced the angiogenesis in granulation tissue, similar to our results (Figs. 2 and 3). SIRT-1 when inhibited, decreased wound healing, while it moderately increased proliferation, suggesting that SIRT-1's role in wound healing is a complex well-coordinated process and proliferation alone is not sufficient for wound repair. Possibly other functions of epidermal SIRT-1 including regulating of cell migration, inflammation and stress response, are the more important determinants for SIRT1's role in wound healing [37].

\subsection{Inflammation, Oxidative Stress, Antioxidants and} Angiogenesis Profiles

The question that emerges from these observations is why natural selection would favor a system whose inflammation is regulated by nutrient availability. In the case of $\mathrm{CR}$, the explanation for this is that immune activation is energetically expensive and increases energy consumption even in nutrient-limited settings [13]. Thus, the inhibitory effect of SIRT-1 on NF- $\mathrm{BB}$ activity is consistent with its role in regulating of available nutrients during times of energy crisis, diverting calories away from the immune system to spare them for essential survival processes [12]. We showed that $\mathrm{R}$ group reduced inflammatory infiltrate, TGF- $\beta 1$, NAG, blood-vessels and VEGF (Fig. 1B, 1C, $1 \mathrm{E}, 1 \mathrm{~F}$ and $1 \mathrm{G}$ ) relation to NR, This corroborates with the findings of Yeung et al. [38] that CR inhibits $\mathrm{NF}-\kappa \mathrm{B}$ expression by reducing inflammation and consequently, the impairment of angiogenesis. On the 2nd day, RC and RL showed lower recruitment of inflammatory infiltrate and TGF- $\beta 1$ than C. On the 7th day, L remained the level of inflammatory infiltrate similar to 2 nd day and higher relation to the other groups, while $\mathrm{C}$ group remained superior TGF- $\beta 1$ relating to other groups and similar to 2nd day (Fig. $2 \mathrm{~B}$ and $2 \mathrm{C}$ ) suggesting the reduction on inflammation in $\mathrm{CR}$ and more evidenced when associated with laser.

Regarding to MPO, RC was higher than other groups on the 2nd day (Fig. 2D). About NAG on the 2nd day, $\mathrm{RC}$ and $\mathrm{L}$ were lower than $\mathrm{C}$, while these were lower than $\mathrm{C}$ and RL on the 14th day (Fig. 2E). The blood-vessel was higher on RL than other groups for all times of follow-up (Fig. 2F). VEGF was more pronunciated on $\mathrm{L}$ and RL on 2nd day than $\mathrm{C}$ and RC. On the 14th day, this expression reduced considerably, 
but the $\mathrm{C}$ group maintained superior to other groups (Fig. 2G). Similar to our finding, lasertherapy has demonstrated its effects on the decrease of inflammatory cells and the improvement of neovascularization. The laser photobiomodulation is able to alternate cellular metabolism, especially by interfering with mitochondrial-membrane potential and ATP synthesis. Furthermore, such modality of laser therapy is also effective in stimulating the cellular cycle and therefore cellular proliferation. Through such capability of biostimulation, laser therapy may induce a decisive impact on the course of biological events that take place during wound healing. The laser treatment depresses the exudative phase (anti-inflammatory effect) at the same time that enhances the proliferative phase during acute and chronic inflammation, activating the local blood circulation and stimulating proliferation of endothelial cells [39].

A

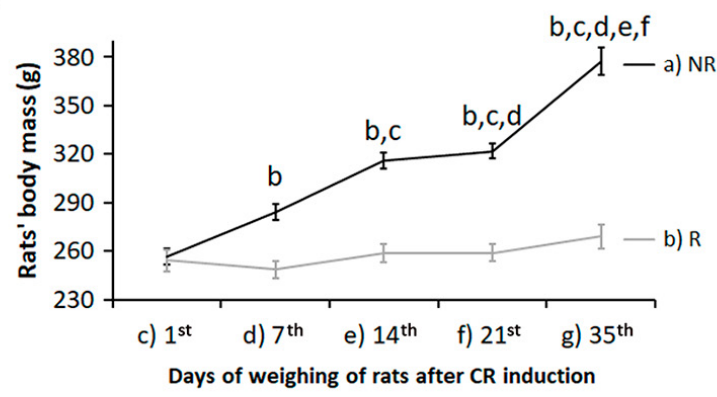

Regarding to oxidative stress, TBARS on 2nd day was lower on $\mathrm{L}$ and $\mathrm{LR}$ relating to $\mathrm{C}$ and $\mathrm{RC}$. However, TBARS on 14th day was higher relating to 2nd day, but RC and RL remain lower than C (Fig. $3 \mathrm{~A})$. About the antioxidants, catalase was more expressed on RL than other groups (Fig. 3C) suggesting the association of $\mathrm{CR}$ and lasertherapy improved antioxidants. About SOD1 and SOD2, the RL group was under expression relation to other groups. Similar to our findings, it is considerable CR might increase the resistance of cells to various types of stress [40]. In this view, CR is associated with increased DNA repair capacity, decreased production of superoxide, and decreased levels of damaged DNA, lipids, and proteins [41].

\subsection{Tissue Formation}

The considerable stimuli of inflammatory phase on 2nd day and modulation of inflammatory response

\section{B}

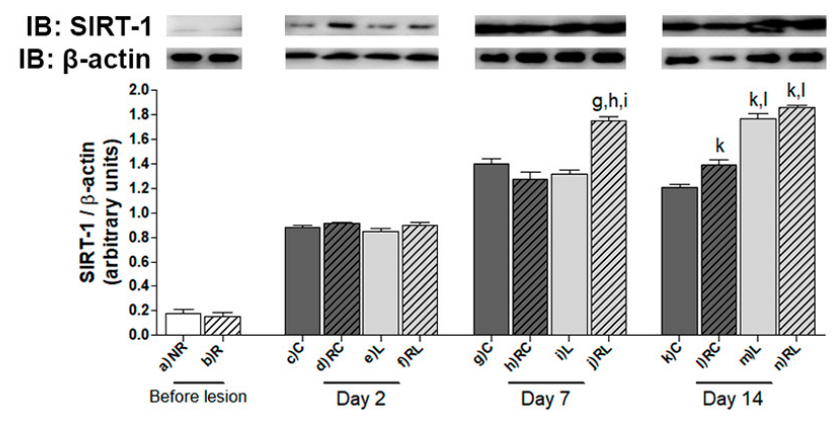

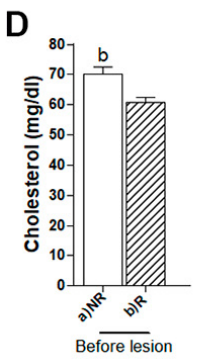
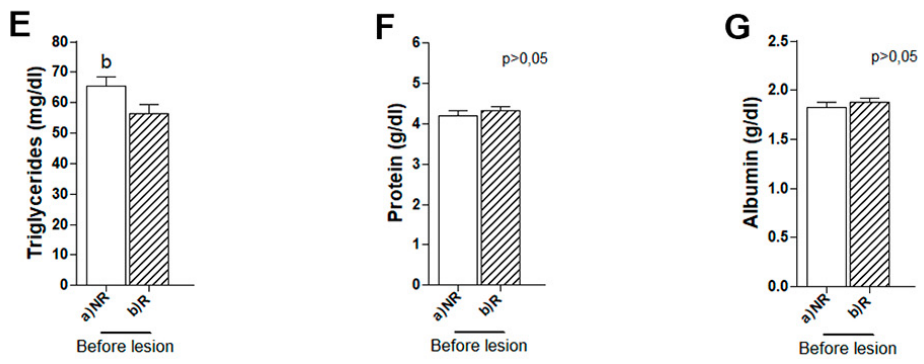

Fig. 1 Caloric restriction confirmation.

(A) Rats' body mass (g) of no-restricted (NR: C/L) and restricted groups (R: CR/LR) during 21 days of $30 \%$ of $\mathrm{CR}(n=16$ animal/group, $t$-Student test); (B) SIRT-1 expression of NR and R groups (before lesion) ( $n=3$ animal/group, $t$-Student test) and on 2nd, 7th and 14th days on groups C (control), RC (restricted control), L (laser) and RL (restricted laser) ( $n=3$ animal/time/treatment, ANOVA Two-way/Tukey post-test). Biochemical analyzes of (C) glucose, (D) cholesterol, (F) protein and (G) albumin $(n=16$ animals/group, Mann-Whitney) of the rats blood serum on 35th day of CR and (E) triglycerides ( $n=16$ animals/group, $t$-Student). Letters in the graph correspond to $p<0.05$ according to the letters in the corresponding group (results in MED \pm SEM). 
A)

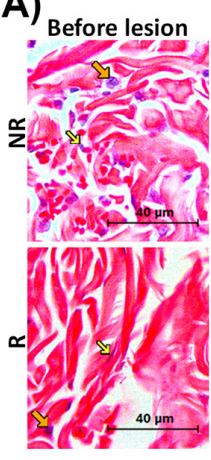

D)

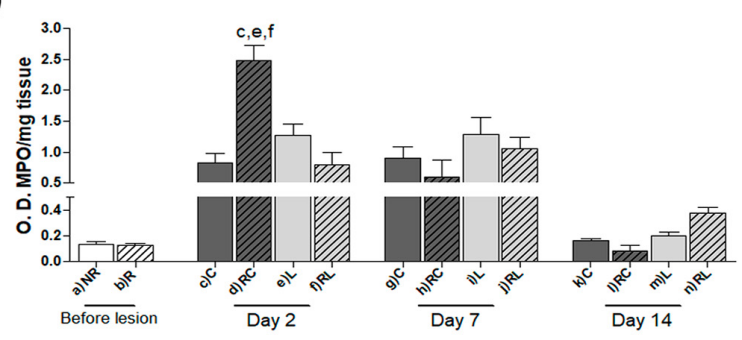

F)

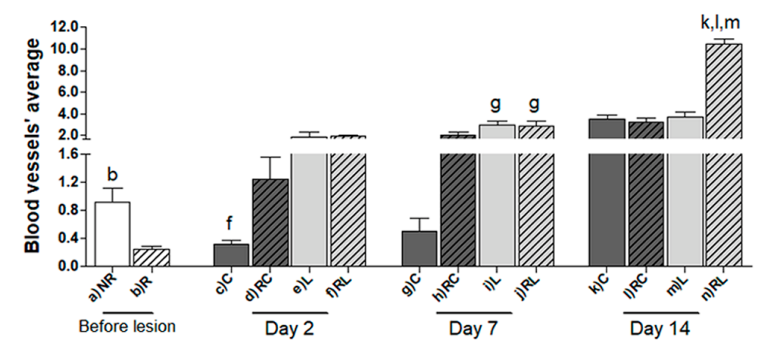

B)

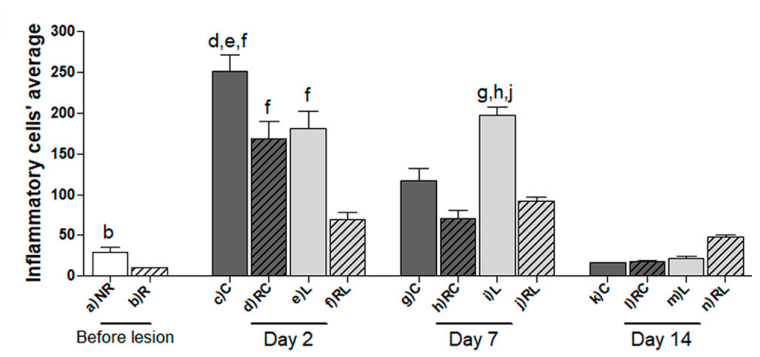

C)

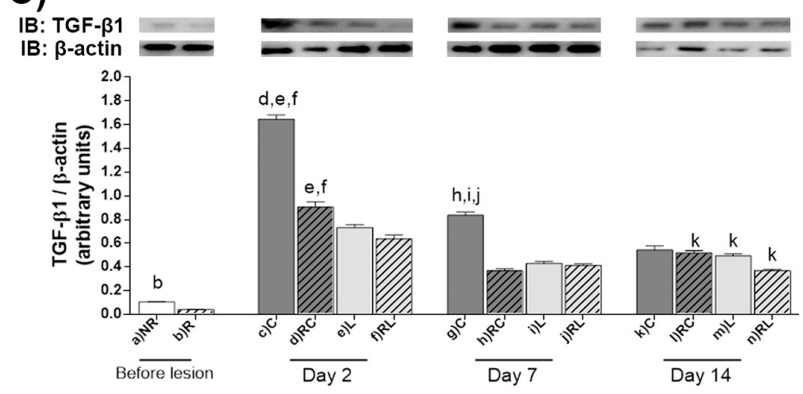

E)

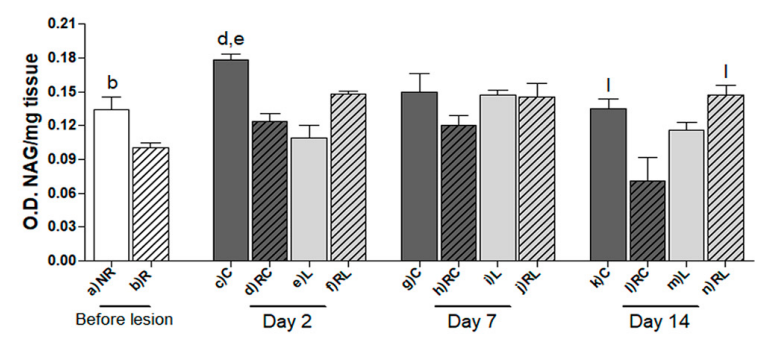

G)

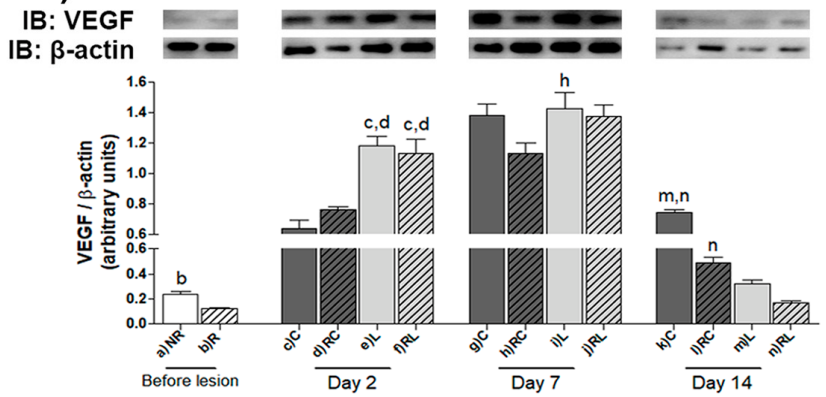

Fig. 2 Inflammation and angiogenic profile.

(A) Representative photomicrograph (HE-400 $\times$ magnification) highlighting the inflammatory infiltrate (orange arrow) and fibroblastos (yellow arrow); (B) Inflammatory cells average; (C) TGF- $\beta 1$ expression; (D) MPO: myeloperoxidase (neutrophils); (E) NAG: N-Acetylglicosaminidase (macrophages); (F) blood-vessels' average and VEGF expression of NR and L (before lesion) $(n=3$ animals/treatment, $t$-Student test) and on 2nd, 7th and 14th days on groups C (control), RC (restricted control), L (laser) and RL (restricted laser) ( $n=3$ animals/time/treatment, ANOVA Two-way/Tukey post-test). Letters in the graph correspond to $p<0.05$ according to the letters in the corresponding group (results in MED \pm SEM). 


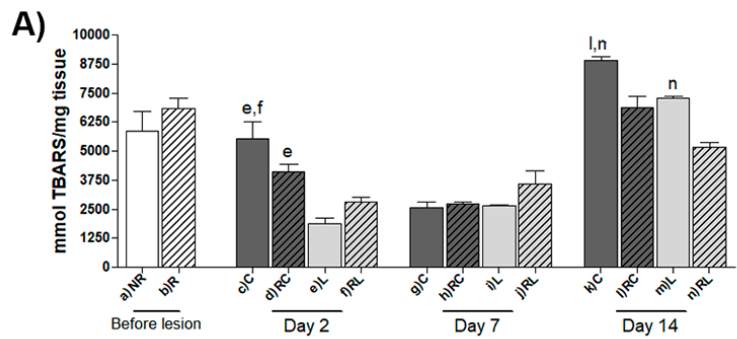

B)

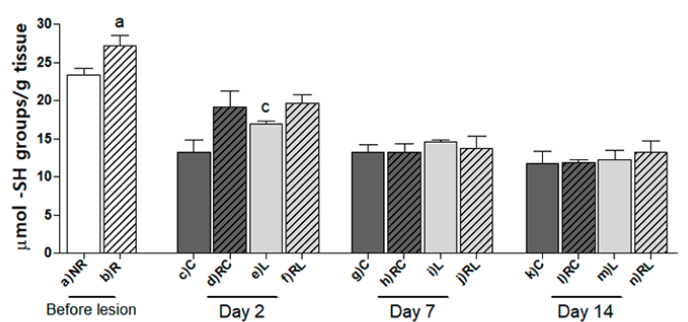

D)

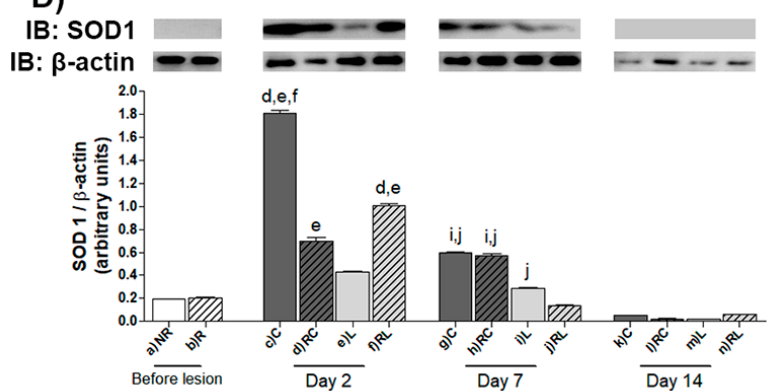

C) IB: Catalase $=-==0$

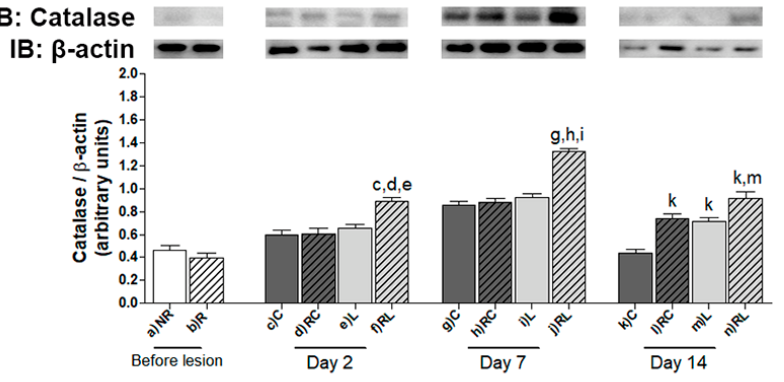

E)

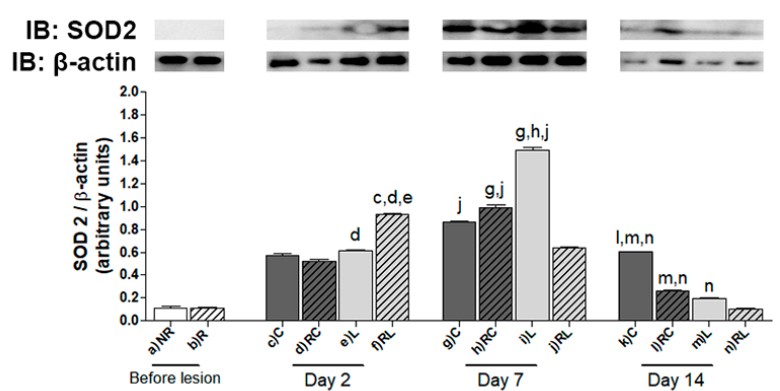

Fig. 3 Oxidative stress and antioxidants.

(A) TBARS; (B) -SH groups, expression of (C) catalase; (D) SOD1; and (E) SOD2 of NR and L (before lesion) ( $n=3$ animals/treatment, $t$-Student test) and on 2nd, 7th and 14th days on groups C (control), RC (restricted control), L (laser) and RL (restricted laser) ( $n=3$ animals/time/treatment, ANOVA Two-way/Tukey post-test). Letters in the graph correspond to $p<0.05$ according to the letters in the corresponding group (results in MED $\pm \mathrm{SEM}$ ).

during the follow-up, associated to oxidative damage control by higher antioxidants production and pronounced angiogenic stimuli, suggest the improvement on reepithelialization on LR superior to $\mathrm{RC}$ and similar to $\mathrm{L}$ and $\mathrm{C}$ (Fig. 4A). The nutritional deficit on RC could have become difficult for the wound healing, but when associated with lasertherapy RL was effective on repair. The CR seems to have also negatively influenced fibroplasia, mainly on RL on 7th and 14th days (Fig. 4B). However, this seems not to influence the collagenesis: RL showed superior HO-Pro than $\mathrm{RC} / \mathrm{L}$ on 7 th day and than $\mathrm{L}$ on 14 th day (Fig. 4C) and superior collagen III/I than other groups (Fig. 4D and 4E).
The scarcity of nutrients on $\mathrm{R}$ was capable to make changes on fibroplasia, HO-Pro and collagen I relation to NR (Fig. 4B-4E). Furthermore, the CR was capable to become the skin (epidermis/dermis) thinner relating to NR (Fig. 4F-4H), suggesting the considerable protein catabolism due to nutrient scarcity [11]. In these ways, CR has shown adverse effects on wound healing and resulted in decreased collagen accumulation and crosslinks [8]. Impaired wound healing was observed in $\mathrm{CR}$ in mice but a short period of ad libitum feeding enhanced their capacity to undergo wound repair. In our study, certainly this wound healing capacity was enhanced by lasertherapy. The cells from CR animals preserved proliferative, 


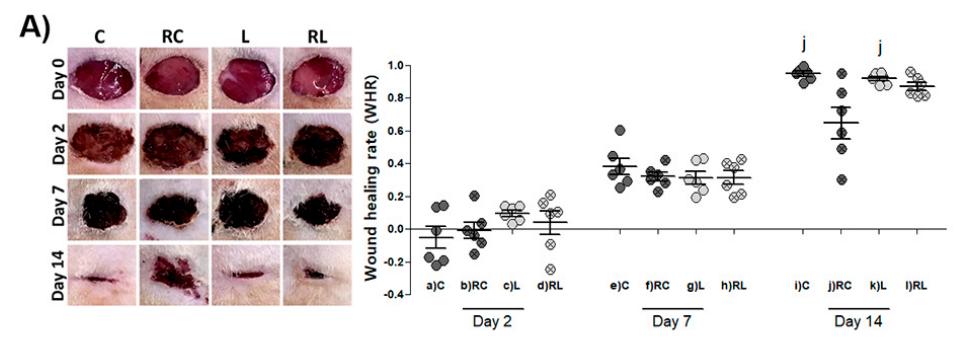

B)

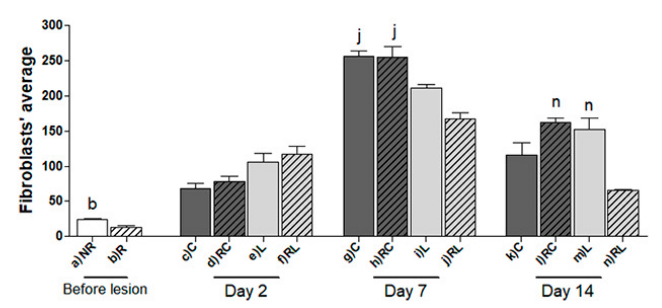

D)

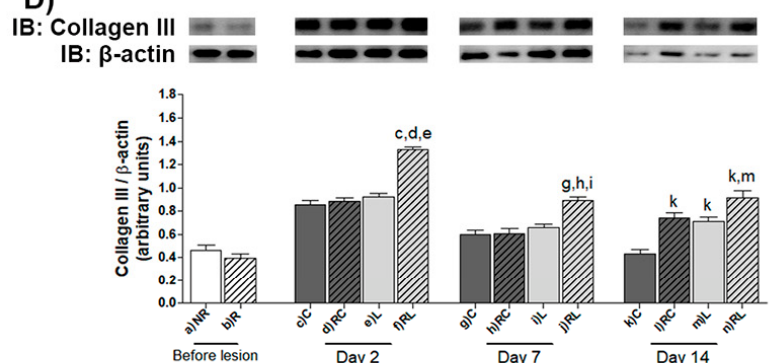

C)

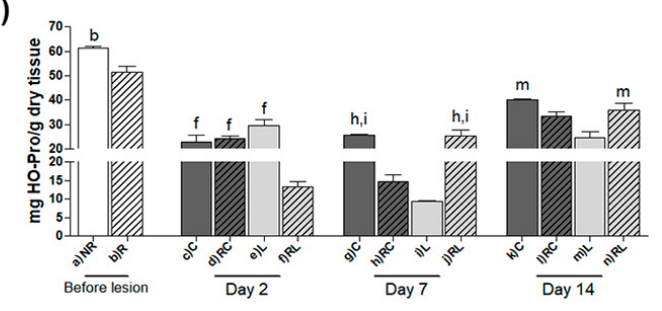

E)
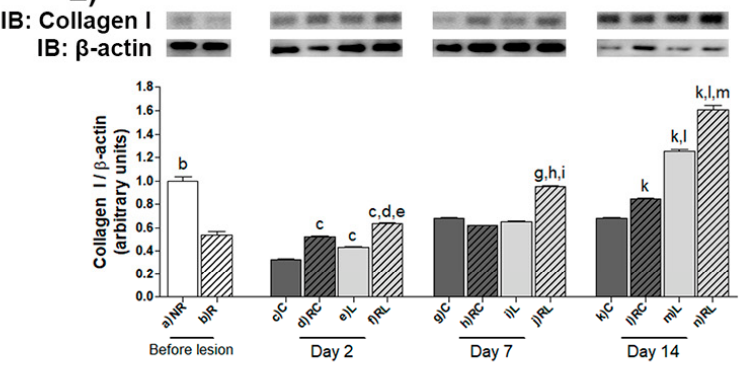

F)

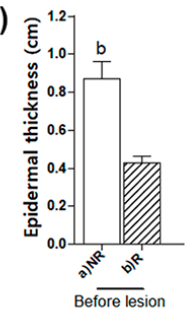

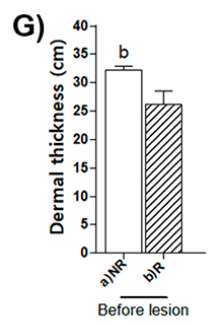

H)

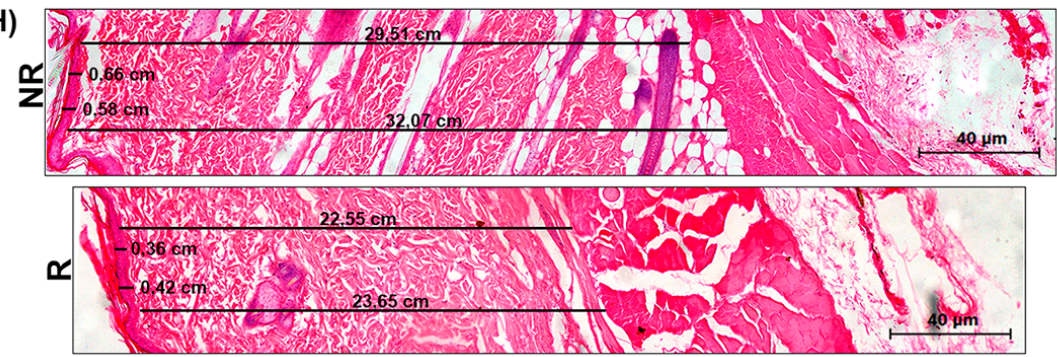

Fig. 4 Tissue formation.

(A) wound healing rate [WHR=(initial area-final area)/initial area]; (B) fibroblasts average; (C) HO-Pro: hydroxyproline, expression of (D) collagen III and (E) collagen I; (F-H) Epidermal and (G-H) dermal thickness of NR and L (before lesion) ( $n=3$ animals/treatment, $t$-Student test) and on 2nd, 7th and 14th days on groups C (control), RC (restricted control), L (laser) and RL (restricted laser) ( $n=3$ animals/time/treatment, ANOVA Two-way/Tukey post-test). Letters in the graph correspond to $p<0.05$ according to the letters in the corresponding group (results in MED \pm SEM).

biosynthetic, and contractile capacities, which was only evident once an adequate source of nutrients became available [42]. Therefore, cell function may be limited by the scarcity of nutrients and limit the growth of granulation tissue.

The use of laser associated with $\mathrm{CR}$ of $30 \%$ was effective in healing, reducing inflammation and controlling oxidative damage by increasing antioxidants. This favored reepithelialization by stimulating the formation of granulation tissue, fibroplasia and collagenesis.

\section{Conclusions}

The $30 \%$ of CR was able to impair the skin (before lesion). Laser therapy has shown to be effective in tissue repair mainly in CR status, being thus the laser 
clinically important strategy to tissue repair in critical situations of CR. Future studies about others clinical situations are important to investigate the action of laser therapy on tissue repair.

\section{Acknowledgements}

To financial support of the Hermínio Ometto Foundation (FHO | UNIARARAS), to undergraduate students Daniela C. Rosa, Danielle S. M. S. Oliveira, Eloísa A. S. Costa, Nathalia M. Lázaro, Marina Viel, Juliana A. Maia, Daniella N. R. Neodini and Leonardo Bagne.

\section{References}

[1] Zeng, R., Lin, C., Lin, Z., Chen, H., Lu, W., Lin, C., et al. 2018. "Approaches to Cutaneous Wound Healing: Basics and Future Directions." Cell Tissue Res. 10: 1-16.

[2] Marreiro, D. D., Cruz, K. J., Morais, J. B., Beserra, J. B., Severo, J. S., and de Oliveira, A. R. 2017. "Zinc and Oxidative Stress: Current Mechanisms.” Antioxidants 6 (2): 24.

[3] Gallego-Muã \pm Oz, P., Ibares-Frã-As, L., Valsero-Blanco, M. C., Cantalapiedra-Rodriguez, R., Merayo-Lloves, J., and Martã-Nez-Garcã-A, M. C. 2017. "Effects of TGF $\beta 1$, PDGF-BB, and bFGF, on Human Corneal Fibroblasts Proliferation and Differentiation during Stromal Repair." Cytokine. 96: 94-101.

[4] Hunt, N. D., Li, G. D., Zhu, M., Miller, M., Levette, A., Chachich, M. E., et al. 2012. "Effect of Calorie Restriction and Refeeding on Skin Wound Healing in the Rat." Age 36: 1453-8.

[5] Speakman, J. R., and Mitchell, S. E. 2011. "Caloric Restrictionm." Mol Aspects Med. 32: 159-221.

[6] Demas, G. E. 2004. "The Energetics of Immunity: A Neuroendocrine Link between Energy Balance and Immune Function." Horm Behav. 45: 173-80.

[7] Ingram, D. K., and de Cabo, R. 2017. "Calorie Restriction in Rodents: Caveats to Consider." Ageing Res. Rev. 39: 15-28.

[8] Reiser, K., Mcgee, C., Rucker, R., and Mcdonald, R. 1995. "Effects of Aging and Caloric Restriction on Extracellular Matrix Biosynthesis in a Model of Injury Repair in Rats." J. Gerontol. A. Biol. Sci. Med. Sci. 50a: B40-7.

[9] Michan, S., and Sinclair, D. 2007. "Sirtuins in Mammals: Insights into Their Biological Function." Biochem J. 404: $1-13$.

[10] Kanfi, Y., Shalman, R., Peshti, V., Pilosof, S. N., Gozlan, Y. M., Pearson, K. J., et al. 2008. "Regulation of SIRT1
Protein Levels by Nutrient Availability.” FEBS Lett. 582: 2417-23.

[11] Gerhart-Hines, Z., Rodgers, J. T., Bare, O., Lerin, C., Kim, S., Mostoslavsky, R., et al. 2007. "Metabolic Control of Muscle Mitochondrial Function and Fatty Acid Oxidation through SIRT1/PGC-1alpha." EMBO J. 26: 1913-23.

[12] Gillum, M. P., Erion, D. M., and Shulman, G. I. 2011. "Sirtuin-1 Regulation of Mammalian Metabolism." Trends Mol Med. 17: 8-13.

[13] Moret, Y., and Schmid-Hempel, P. 2000. "Survival for Immunity: The Price of Immune System Activation for Bumblebee Workers.” Science 290: 1166-8.

[14] Ustaoglu, G., Ercan, E., and Tunali, M. 2017. "Low-Level Laser Therapy in Enhancing Wound Healing and Preserving Tissue Thickness at Free Gingival Graft Donor Sites: A Randomized, Controlled Clinical Study." Photomed Laser Surg. 35: 223-30.

[15] Karu, T. I. 2010. "Mitochondrial Mechanisms of Photobiomodulation in Context of New Data about Multiple Roles of ATP." Photomed Laser Surg. 28: 159-60.

[16] Leite, S. N., Massonmeyers, D. D. S., Leite, M. N., Enwemeka, C. S., and Frade, M. A. C. 2014. "Phototherapy Promotes Healing of Cutaneous Wounds in Undernourished Rats." An. Bras. Dermatol. 89: 899-904.

[17] Taormina, G., and Mirisola, M. G. 2014. "Calorie Restriction in Mammals and Simple Model Organisms." Biomed. Res. Int. 2014: 1-10.

[18] Do Amaral, M. E., Ueno, M., Oliveira, C. A., Borsonello, N. C., Vanzela, E. C., Ribeiro, R. A., et al. 2011. "Reduced Expression of SIRT1 Is Associated with Diminished Glucose-Induced Insulin Secretion in Islets from Calorie-Restricted Rats." J. Nutr. Biochem. 22: 554-9.

[19] Leite, S. N., Jordão Júnior, A. A., Andrade, T. A., Masson, D. S., and Frade, M. A. 2011. "Experimental Models of Malnutrition and Its Effect on Skin Trophism." An. Bras. Dermatol. 86: 681-8.

[20] Caetano, G. F., Frade, M. A., Andrade, T. A., Leite, M. N., Bueno, C. Z., Moraes, A. M., et al. 2015. "Chitosan-Alginate Membranes Accelerate Wound Healing." J. Biomed. Mater. Res. B Appl. Biomater. 103: 1013-22.

[21] Andrade, T. A. M., Masson-Meyers, D. S., Caetano, G. F., Terra, V. A., Ovidio, P. P., Jordão-Júnior, A. A., et al. 2017. "Skin Changes in Streptozotocin-Induced Diabetic Rats." Biochem. Biophys. Res. Commun. 2: 1154-61.

[22] Costa, R., Valente, I., Duarte, D., Rodrigues, J. A., Gomes, P., and Soares, R. 2013. "Xanthohumol Modulates Inflammation, Oxidative Stress, and Angiogenesis in Type 1 Diabetic Rat Skin Wound 
Healing." J. Nat. Prod. 22: 2047-53.

[23] De Aro, A. A., Ferrucci, D. L., Borges, F. P., Stach-Machado, D. R., Macedo, D. V., and Pimentel, E. R. 2014. "Exhaustive Exercise with Different Rest Periods Changes the Collagen Content and MMP-2 Activation on the Calcaneal Tendon." Anat Rec. 297: 281-8.

[24] Buege, J. A., and Aust, S. D. 1978. "Microsomal Lipid Peroxidation." Methods Enzymol. 52: 302-10.

[25] Sedlak, J., and Lindsay, R. H. 1968. "Estimation of Total, Protein-Bound, and Nonprotein Sulfhydryl Groups in Tissue with Ellman's Reagent." Anal Biochem. 25: 192-205.

[26] Sullivan, D. H., Sun, S., and Walls, R. C. 1999. "Protein-Energy Undernutrition among Elderly Hospitalized Patients: A Prospective Study." JAMA 281: 2013-9.

[27] Waitzberg, D. L., Caiaffa, W. T., and Correia, M. I. 2001. "Hospital Malnutrition: The Brazilian National Survey (IBRANUTRI): A Study of 4000 Patients." Nutrition 17: 573-80.

[28] Cowan, D. T., Roberts, J. D., Fitzpatrick, J. M., While, A. E., and Baldwin, J. 2004. "Nutritional Status of Older People in Long Term Care Settings: Current Status and Future Directions." Int. J. Nurs. Stud. 41 (3): 225-37.

[29] Müller, O., and Krawinkel, M. 2005. "Malnutrition and Health in Developing Countries." CMAJ 173: 279-86.

[30] Schaible, U. E., and Kaufmann, S. H. 2007. "Malnutrition and Infection: Complex Mechanisms and Global Impacts." PLoS Med. 4: e115.

[31] Boelsma, E., Lp, V. D. V., Goldbohm, R. A., Klöpping-Ketelaars, I. A., Hendriks, H. F., and Roza, L. 2003. "Human Skin Condition and Its Associations with Nutrient Concentrations in Serum and Diet." Am. J. Clin. Nutr. 77 (2): 348-55.

[32] Shanley, D. P., Aw, D., Manley, N. R., and Palmer, D. B. 2009. "An Evolutionary Perspective on the Mechanisms of Immunosenescence.” Trends Immunol. 30 (7): 374-81.

[33] Dirks, A. J., and Leeuwenburgh, C. 2006. "Caloric Restriction in Humans: Potential Pitfalls and Health Concerns." Mech Ageing Dev. 127: 1-7.

[34] Mechanick, J. I. 2004. "Practical Aspects of Nutritional Support for Wound-Healing Patients.” Am. J. Surg. 188: 52-56.

[35] Chalkiadaki, A., and Guarente, L. 2012. "Sirtuins Mediate Mammalian Metabolic Responses to Nutrient Availability." Nat. Rev. Endocrinol. 17: 287-96.

[36] Kelly, G .S. 2010. "A Review of the Sirtuin System, Its Clinical Implications, and the Potential Role of Dietary Activators like Resveratrol: Part 2." Altern. Med. Rev. 15: 313-28.

[37] Lei, Q., Sample, A., Han, L., Wu, X., and He, Y. Y. 2017. "Epidermal SIRT1 Regulates Inflammation, Cell Migration, and Wound Healing." Sci. Rep. 7 (1): 14110.

[38] Yeung, F., Hoberg, J. E., Ramsey, C. S., Keller, M. D., Jones, D. R., Frye, R. A., et al. 2004. "Modulation of NF-kappaB-Dependent Transcription and Cell Survival by the SIRT1 Deacetylase." EMBO J. 23: 2369-80.

[39] Medrado, A. P., Soares, A. P., Santos, E. T., Reis, S. R., and Andrade, Z. A. 2008. "Influence of Laser Photobiomodulation Upon Connective Tissue Remodeling during Wound Healing." J. Photochem Photobiol B 92 (3): 144-52.

[40] Mattson, M. P. 2008. "Dietary Factors, Hormesis and Health.” Ageing Res Rev. 7: 43-8.

[41] Valko, M., Leibfritz, D., Moncol, J., Cronin, M. T., Mazur, M., and Telser, J. 2007. "Free Radicals and Antioxidants in Normal Physiological Functions and Human Disease.” Int. J. Biochem Cell Biol. 39: 44-84.

[42] Reed, M. J., Penn, P. E., Li, Y., Birnbaum, R., Vernon, R. B., Johnson, T. S., et al. 1996. "Enhanced Cell Proliferation and Biosynthesis Mediate Improved Wound Repair in Refed, Caloric-Restricted Mice.” Mech Ageing Dev. 89 (1): 21-43. 